\title{
Teknokultura
}

ISSNe: $1549-2230$

http://dx.doi.org/10.5209/TEKN.59546

\section{Usos y valoración de Facebook como herramienta para la lucha contra la violencia de género en México}

\author{
Celina Navarro ${ }^{1}$; Luisa Martínez-García²; Simón Vialás Fernández ${ }^{3}$
}

Recibido: 17 de marzo de 2018 / Aceptado: 22 de agosto de 2018 Open peer reviews

Resumen. La violencia de género es un problema global pero con realidades más cruentas en ciertas regiones y países. Este es el caso de México en el que mueren 7 mujeres cada día. Ante este escenario y como respuesta a la incapacidad gubernamental para resolver esta violación constante a los derechos humanos de las mujeres en los últimos años se han realizado diferentes acciones de lucha política para erradicar esta violencia. En algunos casos dinamizadas por las instituciones gubernamentales y mayoritariamente desde la sociedad civil organizada. Partiendo de la concepción de que Internet y las redes sociales son herramientas fundamentales del activismo y de la lucha feminista del siglo XXI, este estudio tiene como objetivo, desde una perspectiva crítica, analizar el uso y valoración que hacen de Facebook las asociaciones que luchan contra la violencia de género en México. A través de una metodología cuantitativa hemos obtenido un mapa descriptivo de las páginas activas de Facebook sobre esta temática. Con la parte cualitativa, realizando entrevistas en profundidad a asociaciones de activistas que usan la red social, hemos identificado las dinámicas de apropiación y usos de la plataforma. Nuestros resultados indican que las entidades usan Facebook de una forma poco crítica pero consiguen tener impacto contra la lucha de género ya que se convierte en una plataforma que crea una noción de sororidad y es percibida como asequible y cercana donde las mujeres se sienten seguras para denunciar casos de violencia.

Palabras clave: activismo; ciberfeminismo; feminicidio; redes sociales; sororidad.

\section{[en] Usages and value of Facebook as a tool for the fight against gender violence in Mexico}

\begin{abstract}
Gender violence is a global problem but with more worrying realities in certain regions and countries. This is the case of Mexico where 7 women die every day. Given this scenario and in response to the government's inability to resolve this constant violation of women's human rights, in recent years different political actions have been carried out to eradicate this violence. In some cases, these are energized by governmental institutions but to a large extent these actions are carried out by organized civil society. Starting from the conception that the Internet and social networks are fundamental tools for the activism in the 21 st century, as well as for feminism, this study aims, from a critical perspective, to analyze the use and appreciation of Facebook by the organizations that fight to eradicate gender violence in Mexico. Through a quantitative methodology we have obtained a descriptive map of the active pages of Facebook on this subject. With the qualitative part, conducting in-depth interviews with activist associations that use the social network, we have identified the dynamics of appropriation and
\end{abstract}

\footnotetext{
1 Universitat Autònoma de Barcelona (España)

e-mail: celina.navarro@uab.cat

2 Universitat Autònoma de Barcelona (España)

e-mail: luisa.martinez@uab.cat

3 Investigador Independiente (España)

e-mail:simon@kinoraw.net
} 
use of the platform. Our results indicate that the entities use Facebook in an uncritical way but they manage to have an impact against gender violence since it becomes a platform that creates a notion of sorority and is perceived as a friendly platform where women feel safe to denounce cases of violence.

Keywords: activism; ciberfeminism; femicide; social networks; sorority.

Sumario. 1. Introducción. 2. Metodología. 3. Resultados. 4. Conclusiones. 5. Referencias

Cómo citar: Navarro, C.; Martínez-García, L.; Vialás Fernández, S. (2018). Usos y valoración de Facebook como herramienta para la lucha contra la violencia de género en México, en Teknokultura 15(2), 285-299.

\section{Introducción}

La violencia de género es un problema global con especial impacto en las sociedades latinoamericanas. La ONU (2017) estima que 14 de los 25 países del mundo con más feminicidios están en América Latina y el Caribe. Según la misma organización, una de cada tres mujeres de estos países sufre violencia física o sexual a lo largo de su vida, lo que la OMS (2013) tipifica de "proporciones epidémicas".

El concepto de violencia de género más usado en los textos académicos y divulgativos es el de la Declaración de las Naciones Unidas sobre la eliminación de la violencia contra las mujeres (1993) y en el Artículo 1 expresa que ésta se entiende como:

... todo acto de violencia basado en la pertenencia al sexo femenino que tenga o pueda tener como resultado un daño o sufrimiento físico, sexual o psicológico para la mujer, así como las amenazas de tales actos, la coacción o la privación arbitraria de la libertad, tanto si se producen en la vida pública como en la vida privada (ONU, 1993, p. 3).

En el contexto mexicano, el Artículo 5, apartado IV, de la 'Ley General de Acceso de las Mujeres a una Vida Libre de Violencia' (2015), expone que se entiende por "Violencia contra las Mujeres: Cualquier acción u omisión, basada en su género, que les cause daño o sufrimiento psicológico, físico, patrimonial, económico, sexual o la muerte tanto en el ámbito privado como en el público" (p. 2). Dicha Ley (2015) pretende coordinar a las entidades federativas y a los municipios para prevenir, sancionar y erradicar la violencia contra las mujeres, así como los principios y modalidades para garantizar su desarrollo y bienestar conforme a los principios de igualdad y de no discriminación. Además, determina la "alerta de género" como una medida para garantizar la seguridad de mujeres y niñas, el cese de la violencia en su contra y/o eliminar las desigualdades producidas por una legislación o política pública que agravia sus derechos humanos (Art.23). La alerta de género, según el Artículo 24, de la misma Ley, se emitirá cuando:

I. Los delitos del orden común contra la vida, la libertad, la integridad y la seguridad de las mujeres, perturben la paz social en un territorio determinado y la sociedad así lo reclame. 
II. Exista un agravio comparado que impida el ejercicio pleno de los derechos humanos de las mujeres.

III. Los organismos de derechos humanos a nivel nacional o de las entidades federativas, los organismos de la sociedad civil y/o los organismos internacionales, así lo soliciten.

(Ley General de Acceso de las Mujeres a una Vida Libre de Violencia, 2015)

Desde la aprobación de dicha Ley se han declarado 13 alertas de género en 12 estados de la república mexicana recopiladas por el Instituto Mexicano de las Mujeres (2018). Por lo anterior, en México la violencia de género es muy preocupante ya que, según datos del Instituto Nacional de Estadística y Geografía (INEGI, 2017), dos terceras partes de las mujeres del país (66.1\%) han sufrido alguna vez en su vida agresiones de tipo sexual, físico, laboral o psicológico. En el mismo informe (INEGI, 2017) se detalla que desde 2007 a 2016 cada cuatro horas muere violentamente una mujer.

En los últimos años, la violencia contra las mujeres está cada vez más presente en la agenda informativa de los medios de comunicación (Menéndez Menéndez, 2014). No obstante, el aumento positivo de la visibilización del problema contrasta con el tratamiento informativo que reciben. Diversos estudios demuestran que los medios de masas ejercen violencia simbólica al incluir en sus contenidos estereotipos machistas y representaciones que devalúan lo femenino (Galarza Fernández, Cobo Bedía y Esquembre Cerdá, 2016; Galtung, 1990). Para contrarrestar esta desigualdad, desde los inicios del movimiento las asociaciones feministas han lanzado sus propios medios especializados, aunque hay que destacar que normalmente solo alcanzan públicos reducidos (Rivero Santamarina y Larrondo Ureta, 2016). Esta tendencia sigue activa y, por ejemplo, en febrero de 2018 se ha abierto la primera radio feminista FM de México (Radio Violeta, 106.1 FM).

Actualmente, las redes sociales se han convertido en otra plataforma de encuentro para los movimientos sociales que les permite saltarse la barrera de los medios de masas (Dahlgran, 2009; Tufecki, 2013). Estas redes han otorgado al movimiento feminista y a la lucha contra la violencia de género nuevas plataformas para llegar a una audiencia potencialmente masiva usando la "autocomunicación de masas" (Castells, 2009). No obstante, hay que tener en cuenta que estas redes se rigen por las dinámicas neoliberales (Caro Castaño, 2015). Entre otros aspectos, las redes tienen una perspectiva operacionalista de buscar las formas de difusión y tipo de contenidos que alcanzarán mayor éxito compitiendo por la atención de los usuarios basándose en el individualismo y las participaciones subjetivas desde el perfil personal (Zajc, 2013). Además, aunque no hay los gatekeepers de los medios tradicionales, los algoritmos de las propias plataformas actúan de filtro infrarrepresentando o sobrerrepresentando temas y perfiles (Caro Castaño, 2015). A pesar de estas dinámicas, algunos autores consideran que los usuarios pueden organizarse para usar las plataformas de una forma diferente y efectiva para sus formas de activismo (Thompson, 2010).

A pesar de la proliferación de los planteamientos críticos sobre el uso de la tecnología, "la 'ceguera de género' ha sido común en los estudios teóricos sobre las tecnologías" (Perdomo Reyes, 2016, p. 1). En este artículo queremos analizar desde una perspectiva crítica el uso que hacen de la red social Facebook las organizaciones 
que luchan para erradicar la violencia de género en México y evaluar si es posible hacer un uso efectivo de la red social para la lucha feminista.

Desde el punto de vista teórico y para hablar del uso activista de la tecnología, desde España se ha difundido el concepto de tecnopolítica a partir del estudio de los movimientos relacionados con el $15 \mathrm{M}$. La tecnopolítica es entendida como el uso estratégico y reflexivo de herramientas digitales para la organización y acción política creando una identidad colectiva (Toret, 2013). Según Lechón Gómez (2015), en los últimos años estamos viviendo en una época en la que la tecnopolítica se ha expresado en movilizaciones como la Primavera Árabe, el Movimiento Passe Livre en Brasil o Wikileaks. Uno de los primeros movimientos en relacionar la tecnología con sus objetivos políticos fue la rebelión zapatista en México que utilizó los medios digitales para conectar sus problemas locales con el mundo global (Lechón Gómez, 2015). No obstante, el Ejército Zapatista de Liberación Nacional (EZLN) no identifica como iniciativa propia la presencia del movimiento en Internet ni en las redes sociales y la atribuye a la sociedad civil organizada (Mariscal, 2013).

En el ámbito concreto de la lucha feminista, el concepto más extendido para hablar del uso de la tecnología desde una perspectiva de género es el ciberfeminismo, aunque engloba perspectivas y corrientes divergentes. Movimientos recientes como el Time's Up, el \#Yotambién (\#MeToo), el movimiento en Iberoamérica \#Niunamenos, sobre todo en Argentina, o la huelga internacional el 8 de Marzo de 2018 han usado Internet y las redes sociales para dar visibilidad a las luchas e intentar crear una sororidad transversal. "La sororidad hace referencia a la hermandad entre mujeres más allá de diferencias de clase, raza, etc." (Varela, 2013, p. 200).

Desde una visión optimista del ciberfeminismo, algunas autoras consideraron que el ciberespacio deja atrás la dicotomía de género y tiene la posibilidad de construir nuevas identidades y relaciones entre individuos. Las autoras más influyentes dentro de esta corriente son Shulamith Firestone (1976) con La dialéctica del sexo, la británica Sadie Plant (1998) con su obra zeros + unos y Donna Haraway (1995) que introdujo la idea del cíborg como ejemplificación del fin del binomio hombre/mujer en el ciberespacio y la creación de identidades alternativas.

Desde una visión contraria, y bajo el marco del tecnofeminismo, se considera que el contexto digital reproduce las estructuras heteropatriarcales de la sociedad (Reverter, 1992). Judy Wajcman (2006) critica el determinismo tecnológico de las autoras anteriores argumentando que los cambios tecnológicos se pueden implantar desde diversas realidades resultando en distintos tipos de relaciones entre los géneros. Este determinismo sitúa a la tecnología fuera de la realidad social y la desvincula del compromiso social de erradicar las desigualdades. Wacjman (2006), por el contrario, afirma que la sociedad y la cultura se influyen mutuamente y, por lo tanto, condicionan su propia evolución.

Sin negar que el ciberespacio puede ofrecer una mayor libertad y fluidez de los géneros, la erradicación de las identidades de mujer y hombre todavía no se ha producido. Por un lado, las grandes empresas de Internet están dirigidas por hombres. Por otro, existe una brecha digital entre clases sociales pero que se acentúa más en el caso de las mujeres. El $13^{\circ}$ Estudio sobre los Hábitos de los Usuarios de Internet en México realizado por la Asociación de Internet.mx 
(2017) refleja cómo el número de personas que usan Internet en México ha mantenido un crecimiento constante hasta alcanzar un $63 \%$ de penetración en 2017 y remarca que no hay diferencias significativas entre sexos. Sin embargo, se deben tener en consideración algunas particularidades de la brecha digital de género, ya que esta no se define únicamente por la capacidad de acceso, sino también por las habilidades y usos. Además, para analizar la brecha digital también habría que tener en consideración otras variables como el nivel educativo o si se encuentra en un área rural o urbana.

Actualmente el ciberfeminismo defiende un uso y apropiación crítica de la tecnología para superar las desigualdades que sufren las mujeres y minorías en el contexto digital (Perdomo Reyes, 2016). La lucha feminista en internet, creando el 'feminismo conectado', tiene también el problema de la exclusión de cierta parte del movimiento, en especial las mujeres mayores o con pocos recursos. Según el estudio empírico de Aristea Fotopoulou (2016) sobre organizaciones feministas de Londres, las mujeres mayores se sienten excluidas de la actual sororidad, ya que ésta se crea en las redes sociales como Facebook y Twitter.

Algunos autores han puesto en duda la formación y el tipo de comunidades en las redes sociales. Remedios Zafra (2010) habla sobre colectividades ligeras donde "la especialización de los individuos (solos) detrás de sus respectivas pantallas (espacios para singularidades y diseños unipersonales) favorece un agrupamiento efímero y sin compromisos" (p. 74). Los activismos en redes sociales, con el individualismo imperante y los algoritmos como filtro activo, tienden a volverse polarizados y aislados (Caro Castaño, 2015; Rendueles, 2013; Zajc, 2013).

Centrándonos en el activismo para lucha contra la violencia de género, es importante remarcar que las desigualdades del mundo real se trasladan al mundo virtual. En base a estas consideraciones, este estudio tiene como objetivo cuestionar la idoneidad de Facebook para tener un impacto real contra la violencia de género y crear sororidad. Con esta base, nos planteamos las siguientes preguntas de investigación:

- ¿Hay un continnum entre el activismo en Facebook contra la violencia de género y el activismo offline?

- ¿Qué uso de Facebook hacen los perfiles que tratan el tema de la violencia de género? ¿Tienen una visión crítica sobre las dinámicas y prestaciones de la plataforma?

- ¿Consigue el activismo crear sororidad en Facebook entre las seguidoras y los seguidores?

- ¿Es Facebook una herramienta útil para luchar contra la violencia de género?

\section{Metodología}

El diseño metodológico de este estudio se divide en dos fases. En la primera se realizó un análisis de contenido cuantitativo de las páginas de Facebook que tienen como uno de sus temas principales luchar contra la violencia de género en México. En la segunda, partiendo de los resultados de la anterior, se realizaron 
entrevistas en profundidad a una submuestra de las organizaciones presentes en la red social.

La muestra cuantitativa está formada por las páginas de Facebook de México que entre sus temáticas tratan la violencia de género. La elección de esta red social se justifica porque es la que cuenta con mayor número de usuarios. Además de estos factores, también hemos optado por centrarnos en este entorno digital por las posibilidades que permite en cuanto a la organización y creación de comunidades en comparación con Twitter (Giraldo-Luque, Núria y Pérez-Arce, 2018).

Hemos obtenido una muestra de 102 páginas de Facebook a partir de una búsqueda especializada en Google durante la primera semana de febrero de 2018 con los siguientes criterios:

1. No estar logueadas en google para evitar sesgo en los resultados en función de las características del perfil personal.

2. Realizando la búsqueda usando los siguientes términos:

a) Palabras clave: violencia, mujer, México

b) Restringirla dentro del dominio "facebook.com"

c) Limitar los resultados a aquellas páginas que han sido actualizadas durante el mes de enero de 2018.

Con el análisis de contenido se busca obtener datos y características generales de las páginas de Facebook para conocer, de una forma general, el uso que se hace de la red y el tipo de contenidos publicados. Para ello, dentro de las variables analizadas se incluyen datos relativos al perfil de las páginas como el título, la temática central, los objetivos, el número de likes o la localización geográfica. Por otra parte, también se codificaron otros datos específicos como la figura jurídica, el tipo de uso de la red, el modo de interacción, la organización de acciones y la construcción de agenda.

Respecto a la parte cualitativa, la herramienta de esta segunda fase es la entrevista en profundidad con la cual queremos obtener el testimonio sobre el uso y valoración que hacen las organizaciones de Facebook para luchar contra la violencia de género. Para elegir a las personas informantes se realizó una selección de las páginas que obtuvimos en la muestra cuantitativa siguiendo criterios de impacto por número de seguidoras y buscando la variedad en el tipo de perfiles de las organizaciones. Se realizaron un total de 7 entrevistas entre mediados de febrero y mediados de marzo de 2018 , todas ellas por videoconferencia y en conjunto sumaron siete horas de testimonios.

\section{Resultados}

\subsection{Mapping de la lucha contra la violencia de género en Facebook}

En México, la mayoría de las comunidades de Facebook que hablan sobre violencia de género están centradas en aspectos generales de la mujer. En menor medida, encontramos 23 que su único tema es la violencia machista contra las mujeres y, con menos recurrencia, encontramos algunas comunidades de la red social con temática LGBT (8) y sobre derechos reproductivos (3). Es importante señalar que no hemos 
encontrado ninguna página que vaya dirigida a minorías étnicas en un país en el que, según el Instituto Nacional para la Evaluación de la Educación (INEE), el 10.45\% de los habitantes son indígenas.

Según el tipo de organización hemos encontrado tres perfiles claramente diferenciados. En primer lugar, hay la presencia de organismos públicos (25), principalmente Institutos de la Mujer a nivel local, estatal y nacional y universidades. El segundo perfil encontrado engloba las entidades civiles (24), que incluyen tanto las páginas de grupos activistas consolidados a nivel online y offline como comunidades que se han creado en Facebook y desarrollan toda su actividad en la red social. Por último, hay presencia de páginas de medios de comunicación y agencias de noticias con perspectiva feminista (8). Esta clasificación nos ha servido para escoger las organizaciones entrevistadas.

El impacto de las páginas analizadas en la muestra varía considerablemente, independientemente del tipo de perfil. Con datos de febrero de 2018, la página con menor alcance tiene 114 likes y la de mayor, 103.075. Esta última corresponde al perfil de Voces Feministas, medio de comunicación entrevistado para este artículo. Dentro de los objetivos descritos en cada página, las palabras que más se repiten, aparte de mujeres y violencia, son derechos (37), género (39), perspectiva (13), promover (14) y políticas (11).

El uso mayoritario que hacen de Facebook las páginas mexicanas que luchan contra la violencia de género es divulgar sus convocatorias de eventos offline (69). En cuanto al uso de la plataforma para generar debate sobre temas de actualidad, solo 34 expresan la opinión de la organización mientras que el resto se limitan a compartir contenidos de otras fuentes. El perfil que más se centra en la construcción de una agenda temática propia es el de los medios de comunicación/agencias de noticias.

A partir de este análisis exploratorio se llevó a cabo una selección de las páginas para la realización de las entrevistas. Con el objetivo de profundizar en el tipo de uso de Facebook por estas organizaciones, escogimos páginas de los tres perfiles principales encontrados (ver Tabla 1). Realizamos una mayor cantidad de entrevistas a páginas con un perfil de medio de comunicación/agencia de noticias porque hacen un uso más activo de Facebook con la creación de agenda temática propia por la lucha de la violencia de género. De este modo, se profundiza más en los usos que hacen de la plataforma.

El alcance geográfico de las organizaciones entrevistadas también es diverso. Por ejemplo, el Instituto Municipal de las Mujeres de Xalapa (IMMX), en Veracruz, y la Fundación Origen, de la Ciudad de México, son de ámbito local. En el caso del medio de comunicación/agencia de noticias Testigo Púrpura, su cobertura es estatal y publica información del Estado de Veracruz. Es pertinente tener el testimonio de dos organizaciones veracruzanas ya que es el único estado de la república mexicana que cuenta con dos alertas de género. Voces Feministas también publica información principalmente estatal, en este caso sobre el estado de Chiapas. Por último, la cobertura de Comunicación e Información de la Mujer (CIMAC), Luchadoras y Ventana pública es de ámbito nacional, pero en el caso de la tercera, que es una radio online, focaliza la información a la ciudad de México. 


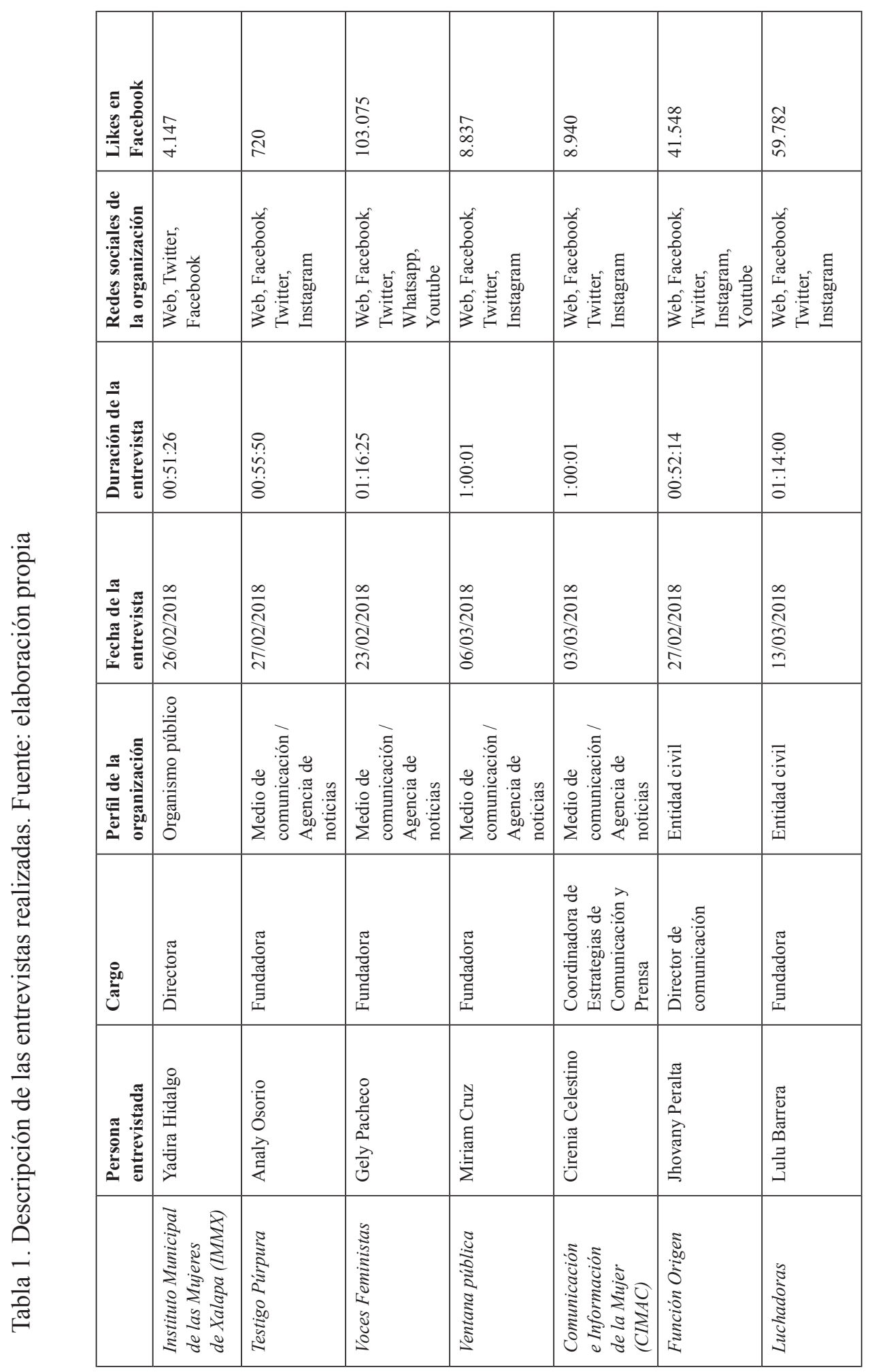




\subsection{Testimonios de las organizaciones que luchan contra la violencia de género}

Facebook es considerada por parte de todas las organizaciones entrevistadas una red social imprescindible para el desarrollo de su actividad debido a su alcance, uso y penetración entre la sociedad mexicana. "La misma dinámica social que hace que todas estemos en las redes sociales hizo que CIMAC tuviera que estar" (CIMAC, comunicación personal, 3 de marzo de 2018). Las personas entrevistadas señalan, además, que los usos que hacen de Facebook responden a objetivos específicos. Por ejemplo, los medios/agencias de noticias utilizan la red social para promocionar las piezas informativas relacionadas con temas de mujeres y violencia y de esta forma intentan generar tráfico hacia su web. Por otra parte, tanto el IMMX como las dos organizaciones civiles Fundación Origen y Luchadoras utilizan Facebook para dar a conocer sus acciones y facilitar información como, por ejemplo, sobre los derechos humanos de las mujeres o violencia de género.

Uno de los puntos fuertes que las entidades destacan de la plataforma son las posibilidades de interacción con los usuarios y otros grupos de Facebook. Todas las organizaciones entrevistadas tienen relaciones con otras entidades a través de la plataforma, no necesariamente centradas en temas sobre mujeres y violencia. Además, estas relaciones llegan incluso, en algunos casos, a convertirse en colaboraciones para la realización de eventos en conjunto (offline) o intercambio de información y técnicas de difusión.

En relación a la interacción con las seguidoras de la página, las respuestas a sus contenidos y el diálogo con los usuarios es una prioridad para las organizaciones. Consideran que el feedback es fundamental para generar comunidad y engagement entre las personas que les siguen. Además, al ser organizaciones que trabajan con temas de violencia de género y por las características de sus publicaciones en Facebook, éstas reciben muchas denuncias de maltrato vía inbox de la misma plataforma o les piden información de qué hacer en caso de violencia de género.

Este flujo de testimonios contiene datos estadísticos que han sido sistematizados por las mismas entidades, en especial por la institución pública $I M M X$, la entidad civil Fundación Origen y los medios CIMAC y Voces Feministas. En el caso de la asociación Luchadoras, ésta ha recibido sobre todo denuncias de violencia digital. De hecho, en el 2017 coordinaron el informe sobre La violencia en linea contra las mujeres en México (Luchadoras, 2017).

Aparte de realizar sus propios informes, en ocasiones las instituciones federales, estatales y/o locales les piden su colaboración para ampliar y contrastar las bases de datos oficiales. "Hay muchas instituciones gubernamentales que se acercan a nosotros para sacar sus notas o sus propias estadísticas. Nosotros somos un banco de información referente" (Fundación Origen, comunicación personal, 17 de febrero de 2018). Los datos que suelen compartir con las instituciones públicas hacen referencia al perfil sociodemográfico de las denunciantes y los tipos de violencia que han sufrido. Esto demuestra la poca capacidad que tiene el gobierno mexicano de registrar fehacientemente los casos y los diferentes tipos de violencia de género y da cuenta de la dimensión de la problemática.

Respecto a las interacciones con los usuarios también hay que destacar la violencia digital, realizada en gran parte por grupos machistas. Las entidades comentan que es escasa y que generalmente se resume en insultos o quejas sobre el servicio dado (como es el caso en el IMMX y la Fundación Origen). No 
obstante, en la mayoría de los casos los mensajes agresivos en Facebook son contestados por los/las seguidores/as generando una dinámica de "defensa", de cuidado entre las personas que conforman el grupo, es decir, una dinámica de sororidad.

En el caso de los medios de comunicación como Voces Feministas y Testigo púrpura estas demostraciones de alianza y de acompañamiento les impulsan a continuar un proyecto que no les genera ningún beneficio económico. "Es como una familia porque los comentarios y la dinámica de Facebook hacen que sea una comunidad cercana" (Voces Feministas, comunicación personal, 23 de febrero de 2018).

Los diferentes tipos de interacción que facilita Facebook también ayudan a las entidades a aumentar el alcance de las publicaciones promocionando sus textos en distintos grupos de la red. Según la temática de cada entrada, ésta se envía a un grupo que congrega a personas que a priori podrían estar interesadas. Además, para promocionar los eventos y/o piezas informativas han recurrido, en algunas ocasiones, al pago de Facebook con el objetivo de generar tráfico e interacciones obteniendo generalmente buenos resultados con esta estrategia. No obstante, no es una fórmula muy utilizada porque representa un gasto y, en ocasiones, un impacto importante en el presupuesto, en especial en las organizaciones que no reciben subvención pública. "No tenemos programado cada cuánto vamos a realizar un pago porque es básicamente cuando nos queda dinero y podemos hacerlo" (Testigo púrpura, comunicación personal, 27 de febrero de 2018).

En referencia a la relación de la página de Facebook con las iniciativas offline, las organizaciones entrevistadas comentan que las promocionan por la red social, en especial la institución pública IMMX. Poniendo otro ejemplo, Testigo Púrpura ha realizado en marzo de 2018 una serie de conferencias con las que da inicio a sus actividades offline y Facebook ha sido una de las plataformas principales para su promoción.

Al mismo tiempo, las actividades offline están pensadas también para paliar la brecha digital y tener más impacto social. En la entrevista con Voces Feministas se habló sobre el lugar que ocupan las mujeres indígenas en su medio/agencia y, al respecto, la fundadora comentó que no hacían distinción entre mujeres y que el tema se trataba pero sin considerarlas como una categoría aparte. Sin embargo, por las características demográficas de Chiapas, ámbito al que pertenece mayoritariamente sus noticias, donde el $14,4 \%$ de la población son mujeres indígenas, considera que esta realidad se debería tomar en cuenta en un futuro, no sin antes hacer un trabajo de contextualización.

La mayoría de la muestra analizada no realiza un discurso muy crítico sobre Facebook, no obstante Ventana Pública, CIMAC y Voces Feministas, los tres medios/agencias, reconocen que los nuevos cambios en el algoritmo de la plataforma han tenido un impacto negativo en el alcance de sus publicaciones. Por su parte, la asociación Luchadoras considera que la red social obedece a los intereses capitalistas patriarcales. Argumenta que les gustaría diseñar una plataforma con menos restricciones y que su interés principal sea el interés común. Esto ha sido planteado en muchos foros feministas pero hasta ahora no se ha materializado. A pesar de las críticas que despierta Facebook para la entidad Luchadoras, esta red social ha sido importante para generar comunidad dentro de sus seguidoras. 


\subsection{Tipo de contenidos compartidos en Facebook}

Como ya hemos visto, la violencia de género es una temática fundamental en todas las organizaciones entrevistadas. "Es necesario visibilizar la violencia, como una violencia contra los derechos humanos y está en gran parte de nuestras publicaciones y de distintas formas" (CIMAC, comunicación personal, 3 de marzo de 2018). Las entidades entrevistadas apuntan que tienen un patrón para las publicaciones en Facebook y que éste lo han diseñado a partir de la observación de las estadísticas y de la evolución orgánica de las publicaciones.

El tratamiento de la violencia de género y el objetivo de estos contenidos es distinto según el perfil de la organización. La institución pública $I M M X$ y la entidad civil Fundación Origen informan a las mujeres sobre la manera de actuar en caso de sufrir violencia de género y, en algunas publicaciones, también apelan a los hombres. En el caso de Luchadoras, éstas siempre tratan el tema de la violencia machista a partir de la exposición de lo que están haciendo las propias mujeres para cambiar esta situación, es decir, visibilizan las acciones de las mujeres.

En los medios de comunicación la perspectiva es distinta siendo las mujeres el objetivo principal de las publicaciones. "No hemos abordado el tema de los agresores, queda siempre como secundario...pero intentamos mantenernos alejados de eso. Las protagonistas siempre son las mujeres" (Testigo Púrpura, comunicación personal, 27 de febrero de 2018). No solo informan sobre el hecho, sobre el qué, sino además sobre el cómo defenderse, cómo denunciar las agresiones y cómo prevenirlas. Para los medios entrevistados esto último es una evolución que ha surgido de manera orgánica en todas sus agendas. Es decir, han valorado de manera cualitativa que aparte de mostrar los casos de violencia de género también es importante dar importancia a cómo prevenir o denunciar estos hechos. "Hacemos un periodismo que contribuya al acceso de las mujeres a los derechos humanos" (CIMAC, comunicación personal, 3 de marzo de 2018). Después de esta evolución todos los medios con perspectiva feminista entrevistados incluyen actualmente información práctica para las mujeres maltratadas.

Los contenidos temáticos de los medios/agencias feministas dependen en gran parte de las piezas informativas de actualidad (noticias, reportajes, entrevistas, etc.) y el número de posts varía en función de éstas y de la promoción que se le quiere dar a los textos. El medio/agencia CIMAC organiza una reunión semanal en la que se diseña las publicaciones para cada red social y los horarios de publicación para obtener mayor impacto. En este caso, por ejemplo, no publican todas las piezas informativas en la red social sino solo las que les interesa promocionar. Por su parte, en Ventana Pública promocionan los diferentes programas que conforman su parrilla programática de su radio online y hacen recordatorios para seguir la emisión en directo.

En contra, la actualidad informativa no dirige en su totalidad la agenda de las publicaciones de las organizaciones civiles ni de la institución pública excepto en los casos de feminicidios. Estas organizaciones publican sobre todo información de sus actividades y promocionan los derechos humanos de las mujeres en diversos ámbitos. En consecuencia, el flujo temático de la información que comparten en Facebook suele alterarse por los eventos organizados offline. El caso de Luchadoras es particular ya que suelen publicar sobre situaciones coyunturales, de actualidad, siendo su principal objetivo visibilizar temas relacionados con las mujeres que no son cubiertos por los medios tradicionales. 
Las publicaciones, además, están marcadas en todas las entidades por el uso del lenguaje con perspectiva de género. "Estamos trabajando en un manual de lenguaje incluyente para toda la administración, aunque es difícil porque no es algo que entre fácil a la gente" (IMMX, comunicación personal, 26 de febrero de 2018). Consideran, además, que el lenguaje de cada red social debe ser diferente y acorde al target de sus seguidoras, pero la característica transversal es que éste debe ser sencillo y claro. El conocimiento sobre el tratamiento del lenguaje lo han adquirido a través de la formación continua y permanente que en algunos casos surge de una necesidad individual y en otras es facilitada por las propias organizaciones.

\section{Conclusiones}

Las redes sociales se han convertido en los últimos años en plataformas para hacer activismo o tecnopolítica (Lechón Gómez, 2015; Toret, 2013). En este estudio hemos analizado el uso que hacen de Facebook organizaciones que luchan en contra de la violencia de género en México. Nuestras conclusiones se deben entender desde una perspectiva crítica de la tecnología y en correlación con el ciberfeminismo actual (Fotopoulou, 2016; Perdomo Reyes, 2016) alejado del determinismo tecnológico y consciente del paralelismo entre las desigualdades de poder en la sociedad y en el mundo online (Galarza Fernández et al., 2016). Esta investigación es un ejercicio teórico que visibiliza el empoderamiento de las mujeres mexicanas para luchar contra la epidemia de la violencia de género desde diferentes trincheras y es, además, una forma de sumarse a las diversas intervenciones políticas que la desactivan (Galarza Fernández et al., 2016).

En el primer mapa descriptivo que hemos elaborado de las páginas de Facebook que trabajan con temáticas relacionadas con la violencia de género en México encontramos tres perfiles de entidades claramente diferenciadas: las instituciones públicas, los medios de comunicación y/o agencias de noticias con perspectiva de género y las entidades civiles que realizan una labor informativa pero que no se consideran medios de comunicación. De forma general, las organizaciones usan Facebook para divulgar sus actividades offline, introducir temas de actualidad relacionados con los derechos humanos de las mujeres y hechos noticiosos sobre violencia de género. Este último uso tiene especial relevancia en los medios/agencias de noticias con el objetivo final de generar tráfico a la web.

Todas las entidades entrevistadas consideran que la violencia ejercida contra las mujeres es una cuestión de derechos humanos por lo que su labor tiene incidencia en el ámbito político de la realidad mexicana a distintas escalas, puesto que sus alcances son distintos (local, estatal, nacional). Los resultados destacan la elevada relación entre las acciones políticas feministas offline y las presentes en Facebook, coherente con las afirmaciones de otros autores sobre la correlación entre activismo offline y online (Sánchez-Duarte y Fernández-Romero, 2017). Este continuum favorece la disminución de las desigualdades por la brecha digital ya que, según estas organizaciones, las actividades y encuentros ayudan a que personas mayores o minorías con dificultades de acceso a Internet puedan participar y sentirse incluidas. No obstante, es preciso seguir trabajando contra la brecha digital.

Uno de los aspectos más valoradas de la red social Facebook, en contraste con otras redes sociales como Twitter, es la posibilidad de interacción y de creación de 
grupos y comunidades. Según las organizaciones, la actividad que llevan a cabo en esta plataforma genera lazos de sororidad entre las mujeres que participan. Esta alianza no solo es evidente en las actividades offline sino también en la propia red social donde las personas suelen identificar y responder de manera orgánica/voluntaria a los comentarios agresivos y/o críticas machistas. Esto provoca que en la mayoría de los casos las organizaciones no tienen que intervenir para defenderse y activar sus protocolos de actuación. Por último, la sororidad que se genera en Facebook es un aliciente y una muestra de apoyo para las personas entrevistadas. Sin embargo, aunque consideran que es una herramienta útil para erradicar este tipo de violencia también son conscientes de que también ha servido como plataforma para el crecimiento de la violencia digital machista.

En referencia a los contenidos publicados en Facebook sobre violencia de género, la visibilización de esta violencia así como la difusión de herramientas para la denuncia son prioritarias incluso para los medios de comunicación/agencias. Al respecto, hemos observado que este planteamiento es una evolución orgánica de estas entidades porque consideran que su rol de informar pasa, también, por ayudar a las mujeres que sufren violencia de género y activar un cambio social para erradicar la violencia contra las mujeres.

Otro de los elementos más destacables es que todas las organizaciones reciben denuncias de violencia de género a través del inbox de la plataforma. Esto demuestra que Facebook es una plataforma asequible y sencilla para que las víctimas de violencia de género puedan denunciar e informarse de las posibilidades a su alcance para salir de su situación. También deja patente el poco impacto que tienen las estrategias gubernamentales para hacer llegar la información a las mujeres sobre cómo actuar en caso de sufrir violencia de género.

La mayoría de los datos estadísticos de las denunciantes son sistematizados por el IMMX, la Fundación Origen, Voces Feministas y Luchadoras y con ellos elaboran sus propios informes sobre la situación de las mujeres que sufren violencia de género en México. En algunos casos, además, comparten la información con otras organizaciones e instituciones públicas (locales, estatales y nacionales).

Para concluir, Facebook es una herramienta útil para la lucha contra la violencia de género y para generar comunidades que organizan actividades colaborativas. Además, la concepción de sororidad que se crea entre los grupos ayuda a crear espacios de encuentro donde las mujeres se sienten seguras para denunciar casos de abusos. Para estas organizaciones Facebook es una red social fundamental para darse a conocer, promocionar sus actividades y/o su labor periodística. El uso que hacen de la plataforma es, en la gran mayoría, poco analítico y reflexivo con las características neoliberales como son la promoción del individualismo y los algoritmos invisibles que resaltan o esconden perfiles y publicaciones según la temática (Caro Castaño, 2015). No obstante, la entidad Luchadoras sí que cuestiona el uso de la plataforma y desearía no formar parte de Facebook aunque, por razones de impacto, tienen un perfil activo.

Finalmente, las principales limitaciones del estudio se hallan en la dificultad de identificar la totalidad de páginas de Facebook activas que se centran en luchar contra la violencia de género en México. Además, las particularidades de la limitación geográfica (México) hace que los resultados no puedan ser extrapolables a otros países. En cualquier caso, ofrecen una información que consideramos valiosa por su originalidad y por la posibilidad de realizar estudios comparativos en un futuro. 


\section{Referencias}

Caro Castaño, L. (2015). Construir y comunicar un "nosotras" feminista desde los medios sociales. Una reflexión acerca del "feminismo del hashtag". Commons: Revista de Comunicación Y Ciudadanía Digital, 4(2), 124.

Castells, M. (2009). Comunicación y poder. Madrid: Alianza.

Dahlgran, P. (2009). Media and political engagement: Citizens, communication, and democracy. Cambridge: Cambridge University Press.

Firestone, S. (1976). La dialéctica del sexo: en defensa de la revolución feminista. Barcelona: Kairós.

Fotopoulou, A. (2016). Digital and networked by default? Women's organisations and the social imaginary of networked feminism. New Media \& Society, 18(6), 989-1005.

Galarza Fernández, E., Cobo Bedía, R., y Esquembre Cerdá, M. (2016). Medios y violencia simbólica contra las mujeres. Revista Latina de Comunicación Social, 71, 818-832. https://doi.org/10.4185/RLCS-2016-1122

Galtung, J. (1990). Cultural Violence. Journal of Peace Research, 27(3), 291-305.

Giraldo-Luque, S., Núria, F.-G., y Pérez-Arce, J.-C. (2018). La centralidad temática de la movilización \#NiUnaMenos en Twitter. El Profesional de La Información, 27(1), 96-105. https://doi.org/10.3145/epi.2018.ene.09

Haraway, D. J. (1995). Ciencia, cyborgs y mujeres: la reinvención de la naturaleza. València: Cátedra.

Instituto Mexicano de las Mujeres (2018, 13 de julio). Alerta de Violencia de Género contra las Mujeres. Recuperado de: https:/www.gob.mx/inmujeres/acciones-y-programas/ alerta-de-violencia-de-genero-contra-las-mujeres-80739

Instituto Nacional de Estadística y Geografía (INEGI) (2017, 18 de agosto). Boletín de Prensa, núm 379/17. Recuperado de: http://www.inegi.org.mx/saladeprensa/boletines/2017/ endireh/endireh2017_08.pdf

Lechón Gómez, D. M. (2015). Snowden nació en la selva Lacandona: reflexiones sobre tecnopolítica y bienes comunes. Teknokultura, 12(3), 577-596.

Luchadoras. (2017). La violencia en línea contra las mujeres en México. Recuperado de: https://sontusdatos.org/wp-content/uploads/2018/01/Informe_ViolenciaEnLinea_ MEXICO_logos-1.pdf

Ley General de Acceso de las Mujeres a una Vida Libre de Violencia (2015). Recuperado de: https://www.gob.mx/cms/uploads/attachment/file/209278/Ley_General_de_Acceso_de_ las_Mujeres_a_una_Vida_Libre_de_Violencia.pdf

Mariscal, Á. (2013, 11 Febrero). El "Subcomandante Marcos" explica el uso de la tecnología del EZLN. Expansión. Recuperado de: https://expansion.mx/nacional/2013/02/11/elsubcomandante-marcos-explica-el-uso-de-la-tecnologia-del-ezln [2018, 5 de marzo]

Menéndez Menéndez, M. I. (2014). Retos periodísticos ante la violencia de género. El caso de la prensa local en España. Comunicación y Sociedad, 22 (julio-diciembre), 53-77.

OMS (2013). Informe de la OMS destaca que la violencia contra la mujer es "un problema de salud global de proporciones epidémicas". Recuperado de: http:/www.who.int/ mediacentre/news/releases/2013/violence_against_women_20130620/es/

ONU (1993). Declaración de las Naciones Unidas sobre la eliminación de la violencia contra las mujeres. Recuperado de: http://www.un.org/ga/search/view_doc.asp?symbol=A/ RES/48/104\&Lang=S

ONU (2017). La regulación del delito de femicidio/feminicidio en América Latina y el Caribe. Recuperado de: http://www.un.org/es/women/endviolence/pdf/reg_del_femicicidio.pdf 
Perdomo Reyes, M. I. (2016). Género y tecnologías. Ciberfeminismos y construcción de la tecnocultura actual. CTS: Revista Iberoamericana de Ciencia, Tecnología y Sociedad, 11.

Plant, S. (1998). Ceros + unos: mujeres digitales + la nueva tecnocultura. Barcelona: Destino.

Rendueles, C. (2013). Sociofobia. El cambio politico en la era de la utopía radical. Madrid: Capitán Swing Libros.

Reverter, S. (1992). Reflexiones en torno al ciberfeminismo. Asparkía, 12, 35-53.

Rivero Santamarina, D., y Larrondo Ureta, A. (2016). La actividad de las publicaciones feministas en red: retos para la transmisión de la cultura feminista en España. Teknokultura, 13(1), 117-140.

Sánchez-Duarte, J.-M., y Fernández-Romero, D. (2017). Subactivismo feminista y repertorios de acción colectiva digitales: prácticas ciberfeministas en Twitter. El Profesional de La Información, 26(5), 894-902.

Thompson, J. B. (2010). Los media y la modernidad: una teoría de los medios de comunicación. Barcelona: Paidós.

Toret, J. (2013). Tecnopolitica: la potencia de las multitudes conectadas. El sistema red $15 M$, un nuevo paradigma de la politica distribuida. Barcelona: Universitat Oberta de Catalunya/Internet Interdisciplinary Institute. Recuperado de: https://tecnopolitica.net/ sites/default/files/1878-5799-3-PB\%20(2).pdf [2018, 9 de febrero]

Tufecki, Z. (2013). "Not This One" Social Movements, the Attention Economy, and Microcelebrity Networked Activism. American Behavioral Scientist, 57(7), 848-870.

Varela, N. (2013). Feminismo para principiantes. Barcelona: B de Bolsillo.

Wajcman, J. (2006). El tecnofeminismo. Madrid: Ediciones Cátedra.

Zafra, R. (2010). Un cuarto propio conectado. (Ciber)espacio y (auto)gestación del yo. Madrid: Fórcola.

Zajc, M. (2013). Social media, prosumption, and dispositives: New mechanisms of the construction of subjectivity. Journal of Consumer Culture, 15(1), 28-47. 